\title{
Circumbinary Planets and the SOLARIS Project
}

\author{
Maciej Konacki ${ }^{1,2}$, Piotr Sybilski ${ }^{1}$, Stanisław K. Kozłowski ${ }^{1}$, \\ Milena Ratajczak ${ }^{1}$ and Krzysztof G. Hełminiak ${ }^{1}$ \\ ${ }^{1}$ Nicolaus Copernicus Astronomical Center, Department of Astrophysics, ul. Rabianska 8, \\ 87-100 Torun, Poland \\ e-mail:maciej@ncac.torun.pl \\ ${ }^{2}$ Astronomical Observatory, Adam Mickiewicz University, ul. Sloneczna 36, 60-286 Poznan, \\ Poland
}

\begin{abstract}
Extrasolar planets in binary and multiple star systems have become a noticeable niche with about 50 planets among over 500 known. Here we however focus on a particular subset of exoplanets in binary star systems - circumbinary planets. They have the unique advantage that a search for circumbinary planets does also significantly contribute to the understanding of their parent stars. We review what is currently known about circumbinary planets and then introduce our two projects aimed at detecting circumbinary planets: The TATOOINE project to find circumbinary planets around non-eclipsing double-lined spectroscopic binary stars with precision radial velocities, and the SOLARIS project to detect circumbinary planets with the timing of eclipses of eclipsing binary stars. For the SOLARIS project, we were granted 2.6 million USD to establish a network of at least four robotic $0.5-\mathrm{m}$ telescopes on three continents (Australia, Africa and South America) to carry out precision photometry of a sample of eclipsing binary stars. We expect that both projects will have a large impact also on the observational stellar astronomy.
\end{abstract}

Keywords. techniques: spectroscopic, photometric, binaries: eclipsing, planetary systems

\section{Introduction}

Searches for planets in close binary systems explore the degree to which stellar multiplicity inhibits or promotes planet formation (Muterspaugh 2005, Muterspaugh et al. 2006, Muterspaugh et al. 2010). Detection of giant planets orbiting both components of short period ( $P<60$ days) binaries ("circumbinary planets") will have significant consequences for theoretical understandings of how giant planets are formed. The binarity of the central body creates an environment in which the evolution of a protoplanetary disk is substantially different than around single stars (Artymowicz \& Lubow, 1994). This must have an effect on the migration of giant planets in a disk as well as on the "parking" mechanism and their final orbit. Likely, also the dynamical interaction between protoplanets and then planets in a multi-planet system should be affected by the central body binarity and presumably result in a different distribution of the orbital elements of planets. Finally, if one assumes that planetary orbits are coplanar with the orbit of an eclipsing binary, then there is an enhanced probability of detecting a circumbinary transiting planet (Konacki 2009a, Ofir 2008, Ofir 2009, Schneider \& Chevreton 1990, Schneider 1994).

Recently, there have been a few claims of detecting circumbinary planets around active eclipsing binary stars using the eclipse timing technique. This is however not the first time when substellar companions or planets have been detected with a timing technique. In addition to the confirmed case of the three rocky planets around a millisecond pulsar 


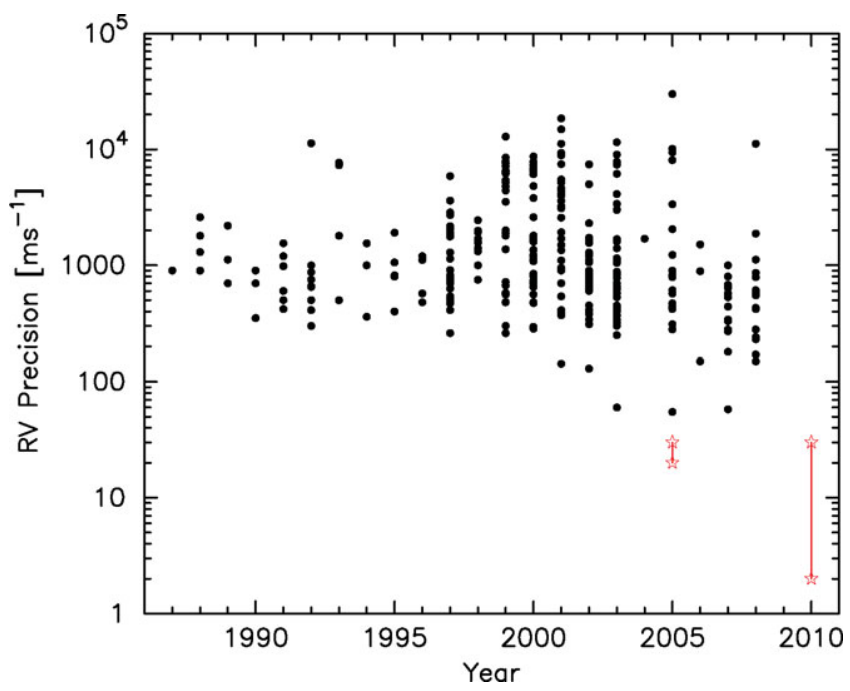

Figure 1. Radial velocity precision (an rms from the orbital fit) for the primaries of double-lined spectroscopic binaries as a function of the publication date based on the ninth catalogue of spectroscopic binary orbits (Pourbaix et al. 2004). It is worth noting that already in the early $\mathrm{XX}$ century an RV precision of several $\mathrm{km} / \mathrm{s}$ for double-lined binaries was possible (Plummer et al. 1908). Current and previous precision range from our method is delimited with the red stars (figure from Konacki et al. 2010).

B1257+12 (Konacki \& Wolszczan 2003), planets have been claimed to orbit a pulsar B0329+54 based on the timing of its radio pulses (Demianski \& Proszynski 1979, Shabanova 1995). Later, it was demonstrated that the timing variation is quasi-periodic and is not due to planets (Konacki et al. 1999). One is left to wonder if these timing variations of active eclipsing binary stars are indeed best explained by a periodic signal due to circumbinary bodies and not an unrecognized quasi-periodic phenomenon (a timing noise) mimicking a periodic planetary signal.

\section{The TATOOINE project - high precision radial velocities of double-lined spectroscopic binary stars}

Radial velocities (RVs) of double-lined spectroscopic binary stars (SB2s) can be effectively used to derive basic parameters of stars if the stars happen to be eclipsing and one can obtain its light curve or their astrometric relative orbit can be determined. It is quite surprising that the RV precision of double-lined binary stars on the average has not improved much over the last 100 years (see Fig. 1). With the exception of our work (Konacki 2005, Konacki 2009a, Konacki et al. 2009b, Konacki et al. 2010), the RV precision for such targets typically varies from $\sim 0.1 \mathrm{~km}^{-1}$ to $\sim 1 \mathrm{~km}^{-1}$ and clearly is much worse than what has been achieved for stars with planets or single-lined binary stars. The main problem with double-lined binary stars is that one has to deal with two sets of superimposed spectral lines whose corresponding radial velocities change considerably with typical amplitudes of $\sim 50-100 \mathrm{kms}^{-1}$. Consequently, a spectrum is highly variable and obviously one cannot measure RVs by noting a simple shift.

We have developed a novel iodine cell based approach that employs a tomographic disentangling of the component spectra of SB2s and allows one to measure RVs of the components of SB2s with the unique precision on the order of 1-10 $\mathrm{m} \mathrm{s}^{-1}$ (Konacki 2009a, Konacki et al. 2009b, Konacki et al. 2010) that is 10 to 100 times more accurate 

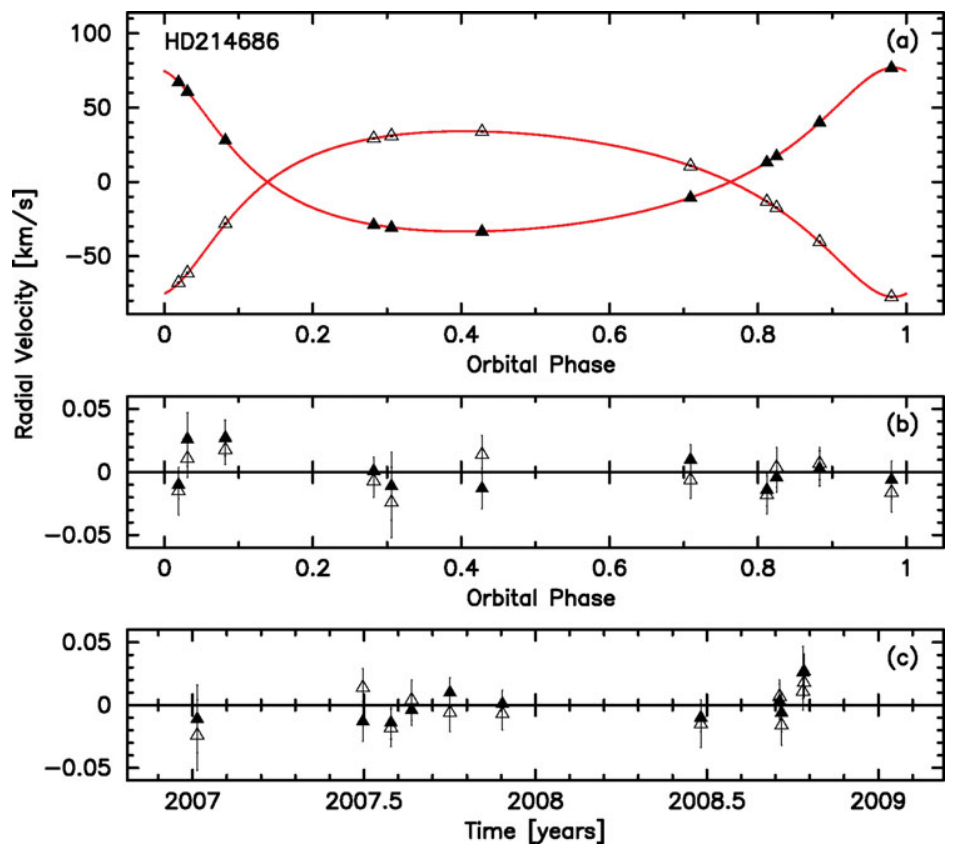

Figure 2. The radial velocities of the spectroscopic binary HD214686 taken with the Shane/CAT (0.9-m !) Hamspec. It is an SB2 with the orbital period of 21.7 days. The 22 measurements (11 for each component) span 2 years (a). The orbital fit residuals are shown as a function of the orbital phase (b) and time (c). The orbital fit is performed simultaneously to the RVs of both components. The rms for the primary is $14.6 \mathrm{~ms}^{-1}$ and $14.7 \mathrm{~ms}^{-1}$ for the secondary (figure from Konacki et al. 2009b).

than archival RVs. Our RV method is described in Konacki (2009a), Konacki et al. (2009b), Konacki et al. (2010).

The idea to search for circumbinary planets around close non-eclipsing double-lined spectroscopic binary stars was born in mid 2003 as a part of our another project (now ended) to search for extrasolar planets in speckle binary star systems with the Keck I/Hires. Over time, it turned out that relatively short period (days to tens of days) double-lined spectroscopic binary stars are actually best suited to measure precision RVs of binary stars as their component's spectra can be disentangled with a tomographic technique.

Our TATOOINE RV project (The Attempt To Observe Outer-planets In Non-singlestellar Enviroments, in collaboration with Matt Muterspaugh) was carried out with the following telescopes/spectrographs: in the years 2003-2007 with the 10-m Keck I/Hires (Keck Obs.), in the years 2006-2007 with the 3.6-m TNG/Sarg (Canary Islands), and in the years 2006-2010 with the 3-m Shane/Hamspec (Lick Obs.). We have also made reconnaissance observations with the 3.9-m AAT/UCLES (Siding Spring Obs.). In a review paper, Udry \& Santos (2007) point out that "circumbinary planets offer a completely unexplored field of investigations." However, our data time span for several SB2s is already 8 years long and the first paper from our effort has been published by Konacki (2009a). This publication describes the first ever limits to circumbinary planets thanks to the precision RVs (see Figs. 2-3). We are currently monitoring close to 50 SB2s (now with HARPS). Among them we have at least one curious case of a possible circumbinary planet. 


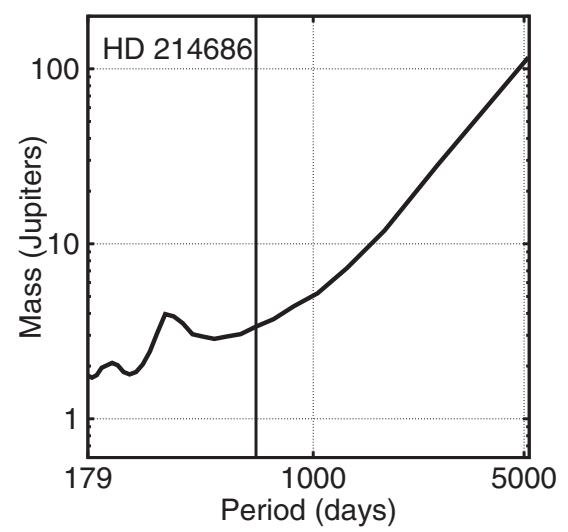

Figure 3. Circumbinary planet detection limits based on RVs from Fig. 2. The planet detection limit was computed using the sophisticated approach of Cumming et al. (1999) (figure from Konacki et al. 2009b).

\section{The SOLARIS project - eclipse timing with automated telescopes}

Accurate light curves of eclipsing binary stars can be used to precisely measure the times of eclipses. Such eclipse timing measurements (ETs) can then be compared with the predicted ones and used to infer information on e.g. the presence of an additional body orbiting the eclipsing binary. The presence of an additional body will cause the movement of the eclipsing binary with respect to the center of mass of the entire system and result in advances/delays in the times of eclipses due to the light time effect. This old idea (it dates back to the 17th century and Ole Roemer) has been used to e.g. detect stellar companions to eclipsing binaries. It can also be used to detect circumbinary planets. However, in order to effectively use ET to detect circumbinary planets from the ground, one needs a massive, well designed and coordinated photometric effort employing several telescopes dedicated to just this task.

Why "Solaris"? Solaris is a novel by an outstanding Polish writer Stanislaw Lem (19212006). The novel is about a circumbinary planet covered with a supposedly conscious ocean. The ocean is studied by humans (with little success) from a station hovering above its surface. Since the novel was for the first time published in 1961, it precedes Star Wars' Tatooine planet (1977) in terms of the first case of a circumbinary planet in pop culture. Solaris was turned into a movie twice: by a great Russian filmmaker Andrei Tarkovsky (1932-1986) and more recently by a Hollywood filmmaker Steven Soderbergh (starring George Clooney).

The SOLARIS project is part of an effort entitled "Eclipsing binary stars as cutting edge laboratories for astrophysics of stellar structure, stellar evolution and planet formation" funded by the European Research Council through a Starting Independent Researcher Grant 2010 awarded to M. Konacki (2010-2015). This grant enables us to establish a global network of four robotic 0.5-m telescopes (Australia, Argentina and South Africa). These telescopes will be used to detect circumbinary planets via eclipse timing and to provide high precision and high cadence light curves for the spectroscopic part of the effort. Such a combination (RV+photometry) will enable us to derive basic parameters of the components of eclipsing binaries with an unprecedented precision (see Fig. 4). The goal of the Starting Grant project is to survey 200 eclipsing binaries using RVs and another 100 (separate sample) eclipsing binaries using eclipse timing to search for circumbinary planets. The spectroscopic part of the project will be carried out with 


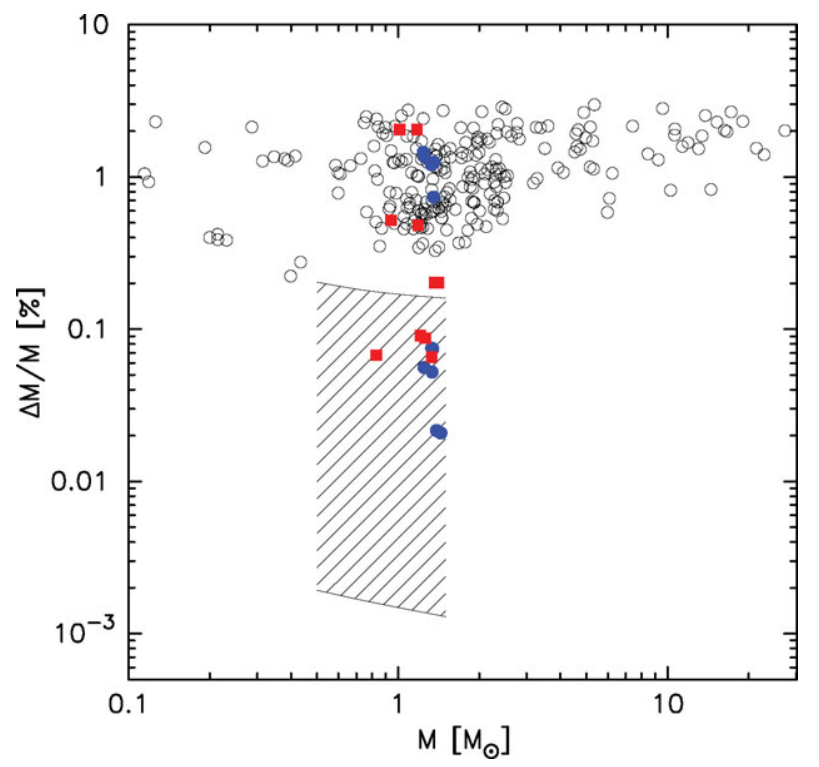

Figure 4. Relative precision in masses for the binary stars with the most accurate mass determinations in the literature. Open circles denote the binaries from a recent review by Torres et al. (2010). Filled (blue) circles denote the double neutron star systems B1913+16, B2127+11C, B1534+12, J0737-3039, J1756-2251 and J1906+0746 characterized with radio pulsar timing (Nice et al. 2008). Filled (red) rectangles denote the masses for HD78418, HD123999, HD200077 and HD210027 from our recent paper by Konacki et al. (2010) and a mass determination for AI Phe from yet another our paper (Helminiak et al 2009). The hatched area is the precision in masses expected to be achieved through this project for double-lined eclipsing binary stars assuming a mass range of the components of $0.5-1.5 \mathrm{M}_{\odot}$, orbital period range of 3-23 days, orbital inclination range of $85-90^{\circ}$, inclination's error range of $0.05-0.3^{\circ}$ and radial velocity amplitude's error range of $1-31 \mathrm{~m} / \mathrm{s}$ (figure from Konacki et al. 2010).

the HRS spectrograph on the 10-m SALT; when HRS becomes available, perhaps from the second half of 2012 .

\section{The impact of the project on the stellar astronomy.}

Modern tests of stellar structure and evolution models require stellar masses accurate to $1 \%$ or better (Torres et al. 2010). Stellar masses are most often derived from the observations of eclipsing binary stars by combining the photometric (light curves) and spectroscopic data (RVs) for double-lined binaries. The relative error in the masses $(\Delta M / M)$ is dominated by the error in the orbital inclination, $i, 3 \Delta i \cos (i) / \sin (i)$, but the errors in the velocity amplitudes obviously also contribute. In particular, when one seeks high precision (below $1 \%$ in the masses). The best results are achieved by targeting eclipsing binaries $(\sin (i) \approx 1$, note the dependence of the error in masses on $i)$. Our RV technique allows us to derive the velocity amplitudes, $K_{1,2}$, of the binary components with a precision of several $\mathrm{ms}^{-1}$ which corresponds to the relative precision $(\Delta K / K)$ of the velocity amplitudes of $0.01 \%$ (the RV amplitudes of SB2s are typically $\sim 50-100$ $k \mathrm{~ms}^{-1}$ ). It easily allows us to derive the mass with a precision up to $0.001 \%$ ! (see Konacki et al. 2010 and Fig. 4). The fact that our stars are eclipsing will hence enable us to combine our precision RVs and our own light curves and determine precise masses, radii and orbital parameters of our targets. 


\section{References}

[1] Artymowicz, P. \& Lubow, S. H. 1994, ApJ, 421, 651

[2] Cumming, A., Marcy, G. W., \& Butler, R. P. 1999, ApJ, 526, 890

[3] Demianski, M. \& Proszynski, M. 1979, Nature, 282, 383

[4] Hełminiak, K. G., Konacki, M., Ratajczak, M., \& Muterspaugh, M. W. 2009, MNRAS, 400, 969

[5] Konacki, M. \& Wolszczan, A. 2003, ApJL, 591, L147

[6] Konacki, M., Lewandowski, W., Wolszczan, A., Doroshenko, O., \& Kramer, M. 1999, ApJ, 519, L81

[7] Konacki, M. 2009a, IAU Symposium, 253, 141

[8] Konacki, M., Muterspaugh, M. W., Kulkarni, S. R., \& Hełlminiak, K. G. 2009b, ApJ, 704, 513

[9] Konacki, M., Muterspaugh, M. W., Kulkarni, S. R., \& Hełminiak, K. G. 2010, ApJ, 719, 1293

[10] Konacki, M. 2005a, ApJ, 626, 431

[11] Muterspaugh, M. W., Konacki, M., Lane, B. F., \& Pfahl, E. 2010, Planets in Binary Star Systems, Astrophysics and Space Science Library, Vol. 366. Berlin: Springer, ISBN: 97890-481-8686-0, 77

[12] Muterspaugh, M. W., Lane, B. F., Kulkarni, S. R., Burke, B. F., Colavita, M. M., \& Shao, M. 2006, ApJ, 653, 1469

[13] Muterspaugh, M. W. 2005, Ph.D. Thesis

[14] Nice, D. J., Stairs, I. H., \& Kasian, L. E. 2008, 40 Years of Pulsars: Millisecond Pulsars, Magnetars and More, 983, 453

[15] Ofir, A. 2009, IAU Symposium, 253, 378

[16] Ofir, A. 2008, MNRAS, 387, 1597

[17] Plummer, H. C. K., Wright, W. H., \& Turner, A. B. 1908, Lick Observatory Bulletin, 5, 21

[18] Pourbaix, D., et al. 2004, A\&A A, 424, 727

[19] Shabanova, T. V. 1995, ApJ, 453, 779

[20] Schneider, J. 1994, Planetary and Space Science, 42, 539

[21] Schneider, J. \& Chevreton, M. 1990, A\&A, 232, 251

[22] Torres, G., Andersen, J., \& Gimenez, A. 2010, A\&AR, 18, 67

[23] Udry, S. \& Santos, N. C. 2007, A\&AR, 45, 397

\section{Discussion}

P. STEE: We know for single stars that there is a strong migration of the planets, especially for giants planets. Do you think that the situation can be similar for planets around double or multiple stars?

M. KonACKI: This is certainly one of the things that we would like to find out thanks to our two surveys.

D. Queloz: I think one second on the timing of the eclipse is very difficult to get on the ground due to the red noise effect.

M. KonACKI: We are aware of this issue. Our simulations suggest that red noise does have an impact on the timing precision but that it is not very serious. We have also made preliminary observations and compared the eclipse timing precision with our simulations and they are consistent.

S. Albrecht: It will be difficult to observe a complete eclipse. Therefore it will be very challenging to get 1 second precision.

M. KonACKI: We will select these detached eclipsing binaries from the quite extensive ASAS catalogue which have possibly short eclipses. 